\title{
A Novel Evidence Theory Based Row Message Passing Algorithm for LDS Systems
}

\author{
Yahui Liu ${ }^{\# 1}$, Jie Zhong ${ }^{\# 2}$, Pei Xiao ${ }^{\& 3}$, and Minjian Zhao ${ }^{\# 4}$ \\ \# College of Information Science and Electronic Engineering, Zhejiang University, Hangzhou, China, 310027 \\ ${ }^{\&}$ Institute for Communication Systems Department of Electronic Engineering, University of Surrey, Guildford, UK \\ ${ }^{1}$ liuyahui@zju.edu.cn, ${ }^{2}$ zhongjie@zju.edu.cn, ${ }^{3}$ p.xiao@surrey.ac.uk, ${ }^{4}$ mjzhao@zju.edu.cn
}

\begin{abstract}
The low density signature (LDS) technique which is able to support massive connectivity with non-orthogonal low density signatures is a promising multiple access (MA) scheme in future 5G systems. We firstly examine the theory of evidence to illustrate the weakness of the update rule for the message passing algorithm in LDS detection process. The combination results against the common sense (RACS) deteriorate the performance of LDS systems with high order modulation schemes considerably. To reduce the occurrence of the RACS, we propose a novel row message passing (RMP) algorithm by gradually updating the a posteriori probability (APP) messages of all variable nodes in each iteration. Hence, the RMP algorithm benefits from the turbo-effect on the rows of indicator matrix which results in fast convergence with moderate complexity. Simulations prove the reduction of iteration times and the effectiveness of the RMP algorithm for LDS systems with high order modulation schemes.

Index Terms-5G multiple access, Low density signature, Massage passing algorithm, Multi-user detection
\end{abstract}

\section{INTRODUCTION}

The low density signature (LDS) structure proposed in [1] is a special case of the code division multiple access (CDMA) sequence design with only a few number of nonzero elements within a large signature length. Therefore, the signature matrix for all users is sparse thus facilitating an affordable multiuser detector with the message passing algorithm (MPA) [2]. Along with the rapid development of the internet of things (IoT), the future fifth generation $(5 \mathrm{G})$ wireless networks are expected to support massive connectivity with a large number of devices, which is the weakness of current long term evolution (LTE) systems. The LDS orthogonal frequency division multiplexing (LDS-OFDF) technique introduced as an uplink multicarrier multiple access (MA) scheme in [3] is able to support a $400 \%$ overloaded condition making it a competitive MA solution for $5 \mathrm{G}$ systems. In [4], the authors constructed a joint sparse graph (JSG), combing the single graphs of LDSOFDM and low density parity check (LDPC) codes. The JSGOFDM system outperforms similar well-known systems such as group-orthogonal multi-carrier CDMA (GO-MC-CDMA) [5] and turbo structured LDS-OFDM [2]. In [6], the sparse code multiple access (SCMA) system is proposed by replacing the the procedure of bit to QAM (quadrature amplitude modulation) symbol mapping and spreading in LDS systems with codebooks. This enables SCMA to benefit from the shaping gain of multi-dimensional constellations [7].

Evidence theory is widely used in many fields such as information fusion and decision-making. In the framework of evidence theory, information fusion relies on the use of the combination rule allowing the belief functions for the different propositions to be combined. The most popular rule of combination is proposed by Dempster [8] [9]. Nevertheless, Dempster's combination rule is a poor solution for the management of the conflict from various information sources [10]. In order to solve this problem, two different kinds of solutions are proposed. One focuses on designing new combination rules [11] and the other pays attention to modifying the evidences before combination [12].

In this contribution, we prove that the update rule of LDS systems is based on Dempster's combination rule. When the chip-messages (or variable-messages) to be combined are highly conflicting, Dempster's combination rule may lead to the result against the common sense (RACS). The RACS deteriorates the performance of LDS systems significantly. Meanwhile, we can observe more obvious deterioration when applying high order modulation. Instead of replacing the combination rule or weighing the evidences which is often the case in the framework of evidence theory, we propose a new "turbo-scheduling" algorithm, namely, the row message passing (RMP) algorithm, to reduce the occurrence of the RACS. To the best of our knowledge, there was no algorithm that reduces the occurrence of the RACS by optimizing the message passing algorithm. The basic idea of the RMP algorithm is to better utilize the updated a posteriori probability (APP) messages. By gradually updating the APP messages of all variable nodes in each iteration, the reliability improves and the convergence rate becomes faster in comparison with the traditional standard message passing (SMP) detector [1].

The rest of the paper is organized as follows. In Section II, we introduce our system model along with the low-complexity belief propagation detector. In Section III, we analyze the update rule of LDS systems based on evidence theory in details. In Section IV, an optimized message passing algorithm is proposed. Finally, the convergence rate and BER (bit error rate) performance are shown by Monte-Carlo simulations in Section V and conclusions are drawn in Section VI.

\section{LDS SYSTEM MODEL}

According to [1], a set of $M$ complex-valued spreading sequences is required to allow $J$ users to transmit their symbols in a shared resource in LDS systems. The LDS system which is a special case of the traditional CDMA system requires that the signature matrix $\mathbf{S}$ contains $d_{c} \ll J$ non-zero 
values on each row and $d_{v} \ll M$ non-zero values in each column. If we replace all the non-zero values in $\mathbf{S}$ with 1 , a regular low density matrix $\mathbf{F}$ is obtained, which is named as the indicator matrix.

For the LDS system, the baseband received signals in one symbol period can be reformed as

$$
y_{m}=\mathbf{h}_{[m]}^{T} \mathbf{x}^{[m]}+v_{m},
$$

where $\mathbf{x}^{[m]}$ and $\mathbf{h}_{[m]}$ denote the combination of symbols transmitted by users participating in chip index $m$ and their corresponding effective receive signature values, respectively. The noise term $v_{m}$ follows Gaussian distribution with zero mean and variance $\sigma^{2}$.

The optimal maximum posterior probability (MAP) detector finds the symbols that maximizes the joint APP

$$
\hat{\mathbf{x}}_{\text {map }}=\underset{\mathbf{x} \in \mathbb{X}^{d_{c}}}{\arg \max } \prod_{m=1}^{M} p\left(\mathbf{x}^{[m]} \mid y_{m}\right),
$$

where $p\left(\mathbf{x}^{[m]} \mid y_{m}\right)$ is the metric of the $m$-th chip for the signal vector $\mathbf{x}$ and $\mathbb{X}$ is the finite signal constellation alphabet.

The LDS structure can be represented by its factor graph in which the transmitted symbols $x_{j}, j=1,2, \cdots, J$ and the chip observations $y_{m}, m=1,2, \cdots, M$ are denoted as variable nodes and chip nodes [2], respectively.

Applying the message passing algorithm to the factor-graph, we can facilitate a suboptimal iterative chip-level multiuser detector. Let $L_{j \rightarrow m}$ and $L_{m \rightarrow j}$ be the log-domain probability sent from variable node $x_{j}$ and chip node $y_{m}$ along the edge $e_{m, j}$. The subsets of indices corresponding to the non-zero locations in the row $m$ and the column $j$ of the signature matrix are denoted as $M(m)$ and $J(j)$, respectively.

The chip-messages are updated by the marginalization function

$$
\begin{aligned}
& L_{m \rightarrow j}(\alpha)=\lambda \max _{\mathbf{x}^{[m]} \in \mathbb{X}^{d_{c}}}^{\star} \\
& x_{j}=\alpha \\
& \left(\sum_{j^{\prime} \in M(m) \backslash j} L_{j^{\prime} \rightarrow m}(\alpha)-\frac{1}{2 \sigma^{2}}\left\|y_{m}-\mathbf{h}_{[m]}^{T} \mathbf{x}^{[m]}\right\|^{2}\right)
\end{aligned}
$$

where $\alpha \in \mathbb{X}, \lambda$ denotes the normalization coefficient and

$$
\max ^{\star}(a, b)=\log \left(e^{a}+e^{b}\right) .
$$

The update of all APP messages can be expressed as

$$
U_{j}(\alpha)=\sum_{m \in J(j)} L_{m \rightarrow j}(\alpha) .
$$

The variable-messages are updated by the extrinsic information

$$
\begin{aligned}
L_{j \rightarrow m}(\alpha) & =\sum_{m^{\prime} \in J(j) \backslash m} L_{m^{\prime} \rightarrow j}(\alpha) \\
& =U_{j}(\alpha)-L_{m \rightarrow j}(\alpha) .
\end{aligned}
$$

Finally, after iteratively applying (3), (5) and (6), the transmitted symbol of user $j$ is estimated as

$$
\hat{x}_{j}=\underset{\alpha \in \mathbb{X}}{\arg \max } U_{j}(\alpha) .
$$

\section{AnAlysis of the Update Rule}

As shown in the last section, we find a potential connection between the message passing process and the evidence theory. More specifically, the update rule in LDS systems is based on Dempster's combination rule. Unfortunately, Dempster's combination rule can result in the RACS when some highly conflicting evidences are combined.

\section{A. Connection Between Evidence Theory and LDS Systems}

Following [8] and [9], let $\Theta$ be the set of $N$ elements corresponding to the $N$ different criminal suspects. This finite set containing $N$ mutually exclusive and exhaustive hypotheses is called the frame of discernment

$$
\Theta=\left\{A_{1}, A_{2}, \cdots, A_{N}\right\} .
$$

The power set of $\Theta$ is the set containing all the possible subsets of $\Theta$, represented by $P(\Theta)$. The $N$ subsets containing only one element are called singletons. Without loss of generality, let the basic probability assignment (BPA) be a function from $\Theta$ to $[0,1]$

$$
f:\left\{\begin{array}{c}
A_{n} \in \Theta \\
f\left(A_{n}\right) \in[0,1],
\end{array}\right.
$$

and which satisfies the following condition

$$
\sum_{A_{n} \in \Theta} f\left(A_{n}\right)=1
$$

Namely, only singletons are assigned with nonzero probability in the framework of evidence theory.

A finite number of BPAs $f_{1}, f_{2}, \cdots, f_{i}$ can be combined to yield a new BPA $f$, using the open world notion of the Dempster's combination rule

$$
\begin{aligned}
f\left(A_{n}\right) & =\sum_{A_{n_{1}} \cap \cdots \cap A_{n_{i}}=A_{n}} f_{1}\left(A_{n_{1}}\right) \cdot f_{2}\left(A_{n_{2}}\right) \cdots f_{i}\left(A_{n_{i}}\right) \\
& =f_{1}\left(A_{n}\right) \cdot f_{2}\left(A_{n}\right) \cdots f_{i}\left(A_{n}\right),
\end{aligned}
$$

where $1 \leqslant n_{i} \leqslant N$ and $f_{i}\left(A_{n_{i}}\right)$ can be regarded as the judgement of witness $i$ towards criminal suspect $A_{n_{i}}$. The total conflict among all the BPAs is defined as

$$
\begin{aligned}
K & =\sum_{A_{n_{1}} \cap \cdots \cap A_{n_{i}}=\Phi} f_{1}\left(A_{n_{1}}\right) \cdot f_{2}\left(A_{n_{2}}\right) \cdots f_{i}\left(A_{n_{i}}\right) \\
& =1-\sum_{n} f\left(A_{n}\right),
\end{aligned}
$$

where $\Phi$ stands for the empty set.

The log-domain notation of (11) is rewritten as

$$
\log \left(f\left(A_{n}\right)\right)=\sum_{i} \log \left(f_{i}\left(A_{n}\right)\right)
$$

Let $\Theta$ be the set corresponding to the constellation alphabet. Regard the natural log-domain messages from chip node $m$ to variable node $j$ as BPA of chip node $m$, i.e., $L_{m \rightarrow j}\left(x_{j}=\alpha\right)=\log \left(f_{i}\left(A_{n}\right)\right)$ with $A_{n}=\alpha$. From (5), (6) and (13), we conclude that the update rule of APP messages and variable-messages is a special case of Dempster's combination rule with only singletons are assigned with nonzero 
probability. Dempster's combination rule is a straightforward way to extend probability theory, but there are some disadvantages. A detailed example is presented in the next subsection.

\section{B. Analysis of the Update Rule}

Example 1 Assuming that BPSK (binary phase shift keying) modulation is applied to the LDS system, i.e., $\Theta=\{+1,-1\}$ and there are three chip nodes connected to variable node $j$, the BPAs of the three chip nodes are denoted as $e_{1}, e_{2}, e_{3}$ in natural log-domain. The combination result of all the three chip nodes is denoted as $e$ which is calculated on the basis of (5) or (13). Detailed information is presented in Table I.

TABLE I

AN EXAMPLE OF UPDATING APP MESSAGE IN LOG-DOMAIN

\begin{tabular}{|c|c|c|}
\hline & $L_{m \rightarrow j}\left(x_{j}=-1\right)$ & $L_{m \rightarrow j}\left(x_{j}=+1\right)$ \\
\hline$e_{1}$ & $\log (0.3)$ & $\log (0.7)$ \\
\hline$e_{2}$ & $\log (0.3)$ & $\log (0.7)$ \\
\hline$e_{3}$ & $\log (1)$ & $\log (0)$ \\
\hline$e$ & -2.4079 & $-\infty$ \\
\hline
\end{tabular}

According to (12), the total conflict among the three chip nodes is $K=1-e^{-2.4079}-e^{-\infty}=0.91$ in this example. The transmitted symbol can never be $x_{j}=+1$ after combination $\left(f\left(x_{j}=+1\right)=0\right)$ because of the third chip node's strong disagreement $\left(f_{3}\left(x_{j}=+1\right)=0\right)$. That is to say, the decision on the transmitted symbol $x_{j}=+1$ is independent of the judgement of the other two chip nodes. This combination result is against the common sense.

To summarize, Dempster's combination rule leads to the RACS when the evidences to be combined are highly conflicting, i.e., $K \rightarrow 1$. And the RACS occurs more frequently with the increase of $K$.

Furthermore, the update rule of chip nodes in (3) can be divided into two parts

$$
\left\{\begin{array}{l}
L_{p}=\sum_{j^{\prime} \in M(m) \backslash j} L_{j^{\prime} \rightarrow m}(\alpha) \\
L_{c}=-\frac{1}{2 \sigma^{2}}\left\|y_{m}-\mathbf{h}_{[m]}^{T} \mathbf{x}^{[m]}\right\|^{2},
\end{array}\right.
$$

where $L_{p}$ and $L_{c}$ denote the a prior information and the channel information, respectively. Obviously, both $L_{p}$ and the addition of $L_{p}+L_{c}$ can be regarded as the combination of evidences as well. The RACS contained in variable-messages will undoubtedly spreads into chip-messages along with the calculation of $L_{p}$. At the same time, the addition of $L_{p}+L_{c}$ itself also leads to the RACS. As the iteration goes on, the RACS spreads into the whole factor graph rapidly and it may cause divergence of the iterative detector.

\section{High Order Modulation}

The situation demonstrated in Table I corresponds to a BPSK modulated LDS system. According to the location of the constellation point $(\mathrm{CP})$, propositions $A_{1}$ and $A_{2}$ are explained as follows

$$
\left\{\begin{array}{l}
A_{1}: \text { The } C P \text { is on the left of axis }-y \\
A_{2}: \text { The } C P \text { is on the right of axis }-y
\end{array} .\right.
$$

According to (11), the combination process of two BPSK modulated message sources corresponding to $e_{1}$ and $e_{2}$ is illustrated in Fig.1. The shaded area represents the conflict between two information sources on the basis of (12).

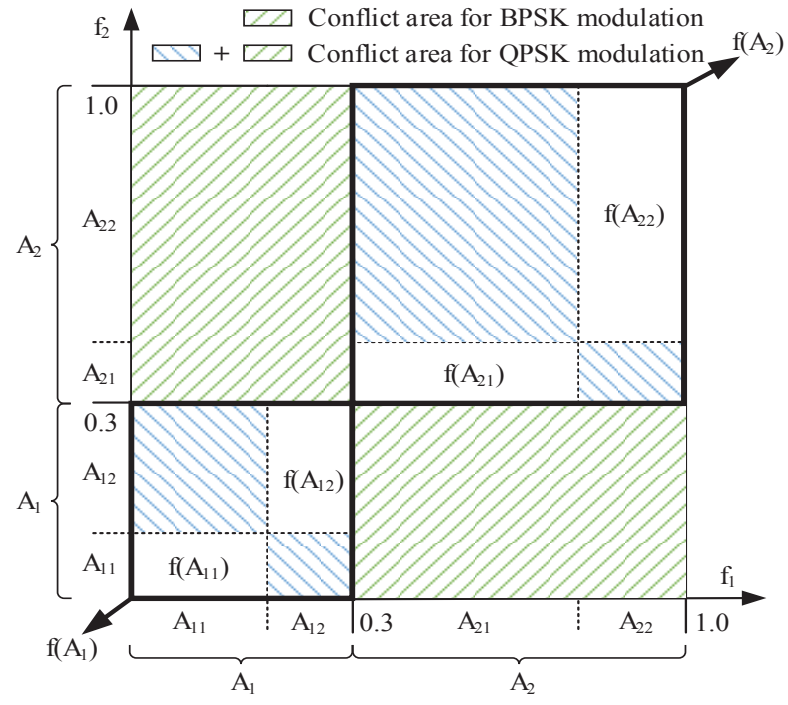

Fig. 1. Representation of combination for two sources.

It is worth pointing out that proposition $A_{1}$ and proposition $A_{2}$ can be divided into a certain number of exclusive and exhaustive assumptions corresponding to the modulation order. For simplicity, we take QPSK (quadrature phase shift keying) modulation as an example. Hence, both proposition $A_{1}$ and proposition $A_{2}$ are divided into two exclusive and exhaustive assumptions

$$
\left\{\begin{array}{l}
A_{1}\left\{\begin{array}{l}
A_{11}: \text { The } C P \text { is in the } 3 r d \text { quadrant } \\
A_{12}: \text { The } C P \text { is in the } 2 \text { nd quadrant }
\end{array}\right. \\
A_{2}\left\{\begin{array}{l}
A_{21}: \text { The } C P \text { is in the } 4 \text { th quadrant } \\
A_{22}: \text { The } C P \text { is in the } 1 \text { st quadrant }
\end{array}\right.
\end{array}\right.
$$

The four propositions $A_{11}, A_{12}, A_{21}$ and $A_{22}$ correspond to a QPSK modulated LDS system. The combination process of two QPSK modulated message sources is illustrated in Fig.1. Obviously, the conflict $K$ becomes larger when the modulation order is higher. As a result, the RACS occurs more frequently in variable-messages and $L_{p}$. We know that the equivalent transmitted signal on $m$-th chip $\mathbf{h}_{[m]}^{T} \mathbf{x}^{[m]}$ has a constellation size $|\mathbb{X}|^{d_{c}}$, where $|\mathbb{X}|$ denotes the cardinality of $\mathbb{X}$. Take $d_{c}=3$ as an example, the equivalent transmitted signal has a constellation size of 8 and 64 for BPSK and QPSK modulated LDS system, respectively. Since the number of the signal constellation points is exponential to $|\mathbb{X}|$, the channel information $L_{c}$ is much less reliable in a high order modulated LDS system. As a conclusion, the RACS occurs more frequently in variable-messages and $L_{p}+L_{c}$ which makes the iterative detector much easier to become divergent in a LDS system with high order modulation schemes.

\section{A New Message Passing Algorithm}

In this section, we propose a novel RMP algorithm. Compared with the traditional SMP algorithm, the new RMP algo- 
rithm reduces the the occurrence of the RACS by updating the APP messages instantly in each loop. Meanwhile, we achieve a faster convergence rate and a moderately low complexity.

\section{A. RMP Algorithm}

The SMP algorithm proposed in [1] is a two-phase (phases $P_{1}, P_{2}$ ) parallel message passing scheme. In phase $P_{1}$, the row processor updates all the chip nodes simultaneously using (3). In phase $P_{2}$, the column processor updates all the variable nodes simultaneously using (5) and (6).

In order to reduce the occurrence of the RACS, we propose a modified approach which is based upon sequential detection. Instead of the separate row-column processing in the SMP algorithm, the proposed RMP algorithm incorporates the calculation of APP messages in the column processing into the row processing. In each iteration, the proposed sequential algorithm employs a row processor to process $M$ rows from the uppermost row to the lowermost row, while no column processor is required. In each row, the row processor reads both APP messages $U_{j}$ and extrinsic messages $L_{j \rightarrow m}$, and then calculates new APP messages $U^{\prime}{ }_{j}$ and new extrinsic messages $L^{\prime}{ }_{j \rightarrow m}$. The APP messages $U_{j}$ are immediately updated, once the chip messages $L_{m \rightarrow j}$ are calculated. Detailed procedure of RMP algorithm is presented in Algorithm 1.

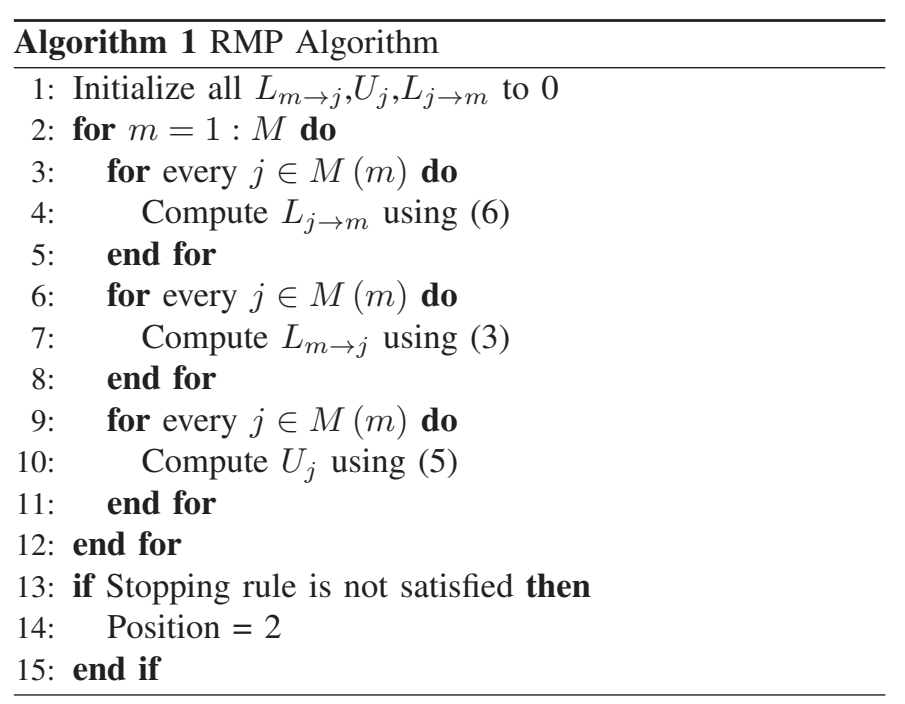

Based on (5) and (6), (5) in RMP algorithm can be simplified by

$$
U_{j}(\alpha)=L_{j \rightarrow m}(\alpha)+L_{m \rightarrow j}(\alpha) .
$$

In the RMP algorithm, chip-messages updated during the current detecting iteration are immediately utilized within the same iteration for calculation of new variable-messages. In other words, the turbo-effect is applied to the update of variable nodes. Thus, the reliability of the variable-messages improves and the convergence rate becomes faster. Since the logdomain probability $U_{j}$ is updated $d_{v}$ times in each iteration, the incorrect APP messages will be corrected or neutralized by the correct chip-messages more quickly. Consequently, the conflict among chip-messages (or variable-messages) are smaller and the likelihood of the RACS is reduced. In this way, the RACS is prevented from spreading into the next iteration.

\section{B. Computational Complexity Per Iteration}

Compared with the update of $L_{m \rightarrow j}$, the computational complexity of updating $L_{j \rightarrow m}$ and $U_{j}$ is negligible. For simplicity, the computational complexity of updating $L_{m \rightarrow j}$ is denoted by the number of max operations, while the complexity of updating $L_{j \rightarrow m}$ and $U_{j}$ is denoted by their updating times in one iteration. Table II presents a list of parameters that compare the computational complexity of the two algorithms.

TABLE II

COMPUTATIONAL COMPLEXITy IN A Single ITERATION

\begin{tabular}{|c|c|c|c|}
\hline & max Operations & $U_{j}$ & $L_{j \rightarrow m}$ \\
\hline $\mathrm{SMP}$ & $M d_{c}\left(|\mathbb{X}|^{d_{c}}-|\mathbb{X}|\right)$ & $J|\mathbb{X}|$ & $J d_{v}|\mathbb{X}|$ \\
\hline $\mathrm{RMP}$ & $M d_{c}\left(|\mathbb{X}|^{d_{c}}-|\mathbb{X}|\right)$ & $M d_{c}|\mathbb{X}|$ & $M d_{c}|\mathbb{X}|$ \\
\hline
\end{tabular}

It is worth noting that $M d_{c}=J d_{v}$. Therefore, the two algorithms has the same number of max operations and $L_{j \rightarrow m}$ updates. The only difference is that the update of $U_{j}$ in RMP algorithm is $d_{v}$ times that of SMP algorithm. Since the update of $U_{j}$ only needs several adders, the overall computational complexity for both algorithms is almost the same.

\section{Simulation Results}

In this section, the robustness to the RACS, the convergence rate and the BER perfermence of the proposed RMP algorithm are compared with the SMP algorithm by Monte-Carlo based simulations over the additive white gaussian noise (AWGN) channel. A regular factor graph structure with 60 chips and 120 users is used. The row weight $d_{c}$ and the column weight $d_{v}$ of the indicator matrix $\mathbf{F}_{60 \times 120}$ are 3 and 6, respectively. The overloading factor is $d_{v} / d_{c}=200 \%$. All simulations have been carried out by using the same spreading signatures for fair comparison. Throughout all the simulations, the overall number of information bits transmitted are equal for both algorithms.

\section{A. Robustness to The RACS}

To analyze the divergence caused by the RACS in the iterative detector, the $E_{b} / N_{0}$ for both algorithms is chosen to ensure the same FER (frame error rate) at the 10-th iteration. In addition, a maximum number of iterations 15 is set to ensure the convergence of the QPSK modulated LDS system. We simulated 5000 frames for each algorithm and their uncoded BER performances at the 10-th and 15-th iteration are shown in the statistical histogram, i.e.,Fig. 2. For SMP algorithm, the more iteration times, the more frames with BER around 0.5. But there are more convergent frames for RMP algorithm as iteration times increase. Furthermore, the RMP algorithm has almost all frames with a BER below 0.25. Namely, the RMP algorithm converges more often and is more robust to the RACS. 


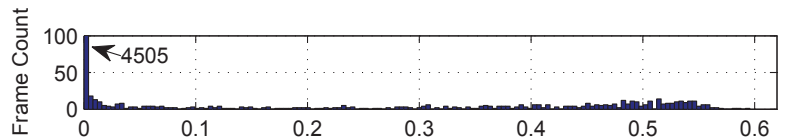

(a) SMP algorithm, uncoded BER per frame at 10-th iteration

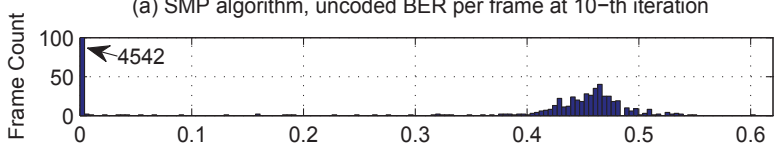

(b) SMP algorithm, uncoded BER per frame at 15-th iteration

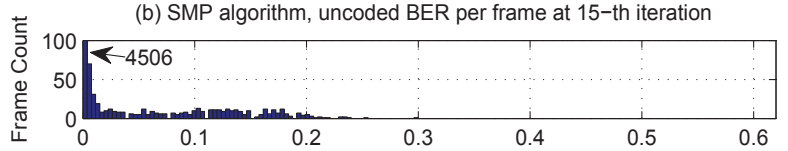

(c) RMP algorithm, uncoded BER per frame at 10-th iteration

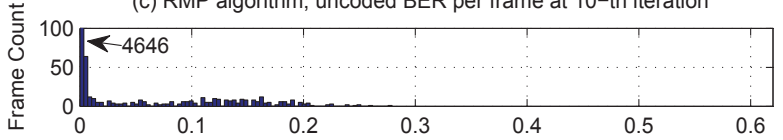

(d) RMP algorithm, uncoded BER per frame at 15-th iteration

Fig. 2. Statistical results of all the 5000 frames over AWGN channel with QPSK modulation for SMP and RMP algorithms. For both algorithms, the maximum number of iterations is set to 15 and the FER is 0.10 at the 10-th iteration. The $E_{b} / N_{0}$ for SMP and RMP algorithm is $12 \mathrm{~dB}$ and $9 \mathrm{~dB}$.

\section{B. Speed of Convergence and BER Performance}

To compare the convergence rate of RMP and SMP algorithms, the maximum number of iterations is set to 3 and 6 for SMP and the maximum number of iterations is set to 3 for RMP in Fig. 3. The RMP detector with a maximum iteration of 3 matches the performance of the SMP detector with a maximum iteration of 6 , therefore the number of iterations for the RMP algorithm is about one half of the required iterations for the SMP algorithm. Hence, the RMP detector exhibits a much faster convergence rate.

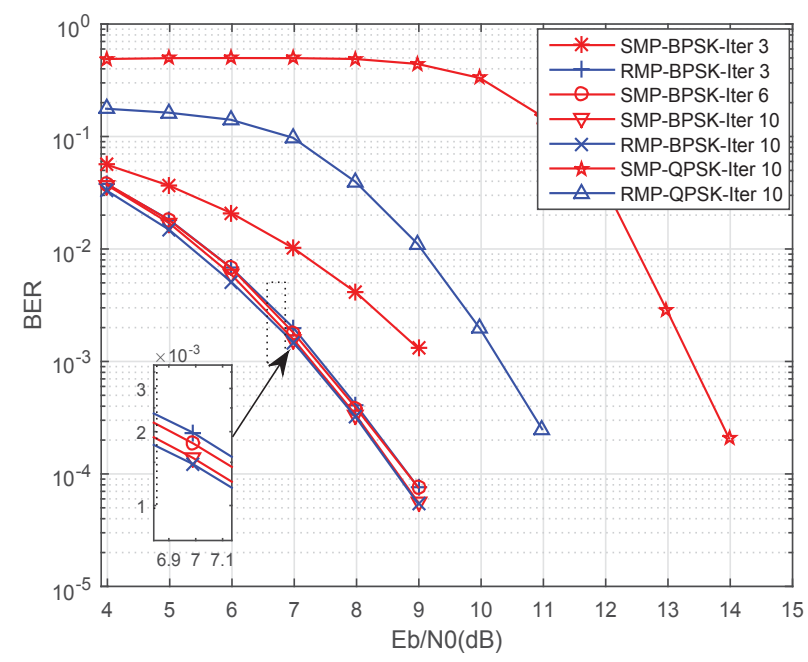

Fig. 3. The convergence rate comparison and performance of the BPSK/QPSK modulated LDS detector over AWGN channel for SMP and RMP algorithms.

In Fig. 3, the other four curves are adopted to compare the uncoded BPSK/QPSK BER performance of SMP and RMP algorithms. The maximum number of iterations is set to 10 for both algorithms. The RMP algorithm outperforms the SMP algorithm slightly when BPSK modulation is employed, while the RMP algorithm brings about $3.0 \mathrm{~dB}$ performance improvement at an uncoded BER $=10^{-3}$ in QPSK modulated systems. The reason is that the RACS appears more frequently in high order modulated LDS systems. The more the RACS appears to be, the better the proposed RMP algorithm detector outperforms.

\section{CONCLUSiON}

In this paper, the update rule of LDS systems has been analyzed based on the theory of evidence. If the high order modulation is applied, the SMP is easily affected by the RACS. By sequentially updating the variable nodes in each iteration, the proposed RMP algorithm improves the reliability of the APP messages and reduces the occurrence of the RACS. Since the APP messages of all variable nodes are updated immediately in each iteration, the RMP algorithm reduces the number of iterations and increases the convergence rate of the detection process. The complexity per iteration for both algorithms is almost the same, resulting in a lower total complexity for the RMP algorithm. Simulations prove that the RMP algorithm reduces the likelihood of the RACS and achieves a much better BER performance.

\section{ACKNOWLEDGEMENT}

This work was sponsored by the National Science Foundation of China (NSFC) under Grant 61201230, the Fundamental Research Funds for the Central Universities, and the National High Technology Research and Development Program (863 Program) of China under Grant 2014AA01A707.

\section{REFERENCES}

[1] R.Hoshyar, F.P.Wathan, R.Tafazolli, "Novel Low-Density Signature for Synchronous CDMA Systems Over AWGN Channel," Signal Processing, IEEE Transactions on, vol.56, no.4, pp. 1616-1626, April. 2008.

[2] R.Razavi, M.Al-Imari, M.A.Imran, R.Hoshyar, D.Chen, "On Receiver Design for Uplink Low Density Signature OFDM (LDS-OFDM),' Communications, IEEE Transactions on, vol.60, no.11, pp. 3499-3508, November. 2012.

[3] R.Hoshyar, R.Razavi, M.Al-Imari, "LDS-OFDM an Efficient Multiple Access Technique," Vehicular Technology Conference (VTC 2010-Spring), 2010 IEEE 71st, pp. 1-5, May. 2010.

[4] L.Wen, R.Razavi, M.A.Imran, P.Xiao, "Design of Joint Sparse Graph for OFDM System," Wireless Communications, IEEE Transactions on, vol.14, no.4, pp. 1823-1836, April. 2015.

[5] F.Riera-Palou, G.Femenias, J.Ramis, "On the design of uplink and downlink group-orthogonal multicarrier wireless systems," Communications, IEEE Transactions on, vol.56, no.10, pp. 1656-1665, October. 2008.

[6] Nikopour Hosein, Baligh, Hadi, "Sparse code multiple access," Personal Indoor and Mobile Radio Communications (PIMRC), 2013 IEEE 24th International Symposium on, pp. 332-336, Sept. 2013.

[7] M.Taherzadeh, H.Nikopour, A.Bayesteh, H.Baligh, "SCMA Codebook Design," Vehicular Technology Conference (VTC Fall), 2014 IEEE 80th, pp.1-5, Sept. 2014.

[8] A.P. Dempster, "Upper and lower probabilities induced by a multivalued mapping." The annals of mathematical statistics., pp. 325-339, 1967.

[9] G. Shafer, A mathematical theory of evidence. Vol. 1. Princeton: Princeton university press, 1976.

[10] L.A. Zadeh, "Review of a mathematical theory of evidence." AI magazine, pp. 81-83, 1984.

[11] E. Lefevre, O. Colot, P. Vannoorenberghe, "Belief function combination and conflict management." Information fusion, pp.149-162, 2002.

[12] Yang Y, Han D, Han C, "Discounted combination of unreliable evidence using degree of disagreement." International Journal of Approximate Reasoning, pp. 1197-1216, 2013. 